\title{
Sign and Amplitude Representation of the Forex Networks
}

\author{
S. GworeK ${ }^{a}$, J. Kwapié̃ ${ }^{a}$ And S. DrożD $\dot{Z}^{a, b}$ \\ ${ }^{a}$ Institute of Nuclear Physics, Polish Academy of Sciences, E. Radzikowskiego 152, 31-342 Kraków, Poland \\ ${ }^{b}$ Faculty of Mathematics and Natural Sciences, University of Rzeszów, Rzeszów, Poland
}

\begin{abstract}
We decompose the exchange rates returns of 38 currencies (including gold) into their sign and amplitude components. Then we group together all exchange rates with a common base currency, construct Minimal Spanning Trees for each group independently, and analyze properties of these trees. We show that both the sign and the amplitude time series have similar correlation properties as far as the core network structure is concerned. There exist however interesting peripheral differences that may open a new perspective to view the Forex dynamics.
\end{abstract}

PACS numbers: 89.75.Da, 89.75.Fb

\section{Introduction}

A series of recent papers [1-4] shows that the currency exchange market has interesting properties if expressed as a network in different representations corresponding to different base currencies. Each representation is defined by a set of exchange rates sharing a given base which can form, via their cross-correlations, a binary or weighted network. Depending on a particular choice of the base, the network displays a hierarchy with clusters of coupled currencies, with one or a few hubs and many peripheral nodes, among which a scale-free order can be observed. A node of the highest centrality is USD, which attracts a number of satellite currencies due to strong economical ties between the corresponding countries or explicit currency pegs. However, a careful inspection of the temporal evolution of the currency network, one observes a clear long-term trend according to which the USD node gradually loses its strength giving more freedom to its previously coupled neighbours. By studying the temporal stability of the network structure in different representations we showed that the only major currency which gains some importance owing to this trend is EUR [3].

In the present paper we take a closer look at some subtleties of the currency couplings. We study time series of daily exchange rates for a set of $N=38$ free-convertible currencies and gold [5]. Our data spans a decade-long interval from Jan 1, 1999 to Dec 31, 2008 ( $T=2519$ trading days) and consists of all possible $N(N-1)=1406$ combinations of the exchange rates $B / X \equiv \Gamma_{X}^{B}$, where $B$ is called the base currency (this rate expresses how many units of $X$ one needs to buy 1 unit of $B$ ). The exchange rates $\Gamma_{X}^{B}(i), i=1, \ldots, T$ obey the two fundamental relations:

$$
\begin{aligned}
& \Gamma_{X}^{Y}(i)=1 / \Gamma_{Y}^{X}(i), \\
& \Gamma_{X}^{Y}(i)=\Gamma_{Z}^{Y}(i) \Gamma_{X}^{Z}(i) .
\end{aligned}
$$

which reduce the effective dimensionality of the phase space. The second relation is called the triangle rule. In both relations we neglected the transaction costs. As usual in this type of analysis, due to strong nonstationarity of the exchange rates, we consider their logarithmic increments

$$
g_{X}^{B}(i)=\ln \Gamma_{X}^{B}(i+1)-\ln \Gamma_{X}^{B}(i) .
$$

Each time series of $g_{X}^{B}(i)$ was preprocessed to remove possible artifacts. A filter was also used in order to remove exceptionally high values which could influence outcomes of numerical analysis. Those returns whose absolute value exceeded a threshold $p=10 \sigma$ ( $\sigma$ denoting standard deviation of the time series) were replaced by $\pm p$.

We want to avoid constructing of a single network from all the exchange rates since such a network would be large, information-overloaded, and difficult to comprehend (even though this kind of study can in principle be carried out - e.g. [6]). Therefore we divide the whole set of time series into much smaller subsets consisting of the exchange rates with a given base currency $B$. An advantage of this approach is that by fixing $B$ and considering only the rates $B / X$ we effectively eliminate this currency from an analysis. As a consequence, instead of the less convenient exchange rates, we can speak of the individual currencies and the relations between them.

For a given subset of (normalized) time series, we construct an $(N-1) \times T$ data matrix $\boldsymbol{M}^{B}$ and then calculate the correlation matrix $\boldsymbol{R}^{B}$ :

$$
\boldsymbol{R}_{X Y}^{B}=\frac{1}{T} \boldsymbol{M}_{X}^{B} \tilde{\boldsymbol{M}}_{Y}^{B}
$$

where tilde stands for matrix transpose. Matrix elements $R_{X Y}^{B}$ are the correlation coefficients calculated for a pair of rates $B / X$ and $B / Y$ and can be considered a measure of couplings between currencies $X$ and $Y$ in the $B$-based representation of the market. By construction, $R_{X Y}^{B}=R_{Y X}^{B}$. The matrix $\boldsymbol{R}^{B}$ completely defines the structure of an undirected network, in which currencies, 
expressed in terms of $B$, are nodes and the matrix elements are connection weights $\omega_{X Y}^{B}=\left|R_{X Y}^{B}\right|$.

Such a network can be visualized as a complete graph, but this would not be an optimal graphical representation in our case of fully connected networks with $N-1=37$ nodes and $(N-1)(N-2) / 2=666$ internode links. Instead, we prefer to consider a minimal spanning tree (MST) [7] - a subset of the whole network, consisting of all $N-1$ nodes and only $N-2$ links. In order to calculate MST, we change the correlation matrix into a distance matrix $\boldsymbol{D}^{B}$ with elements defined by the metric:

$$
d_{X Y}^{B}=\sqrt{2\left(1-R_{X Y}^{B}\right)} .
$$

In general, $0 \leq d_{X Y}^{B} \leq 2$; special cases are: $d_{X Y}^{B}=0$ for identical signals, $d_{X Y}^{B}=\sqrt{2}$ for uncorrelated signals (in the limit $T \rightarrow \infty$ ), and $d_{X Y}^{B}=2$ for signals opposite in phase. Based on the distance matrix $\boldsymbol{D}^{B}$, MST is created by sorting its elements from the largest to the smallest, and connecting the nodes with links according to this ranking in such a manner that each pair of nodes may be connected only via a single path (no cycles allowed). This assures the dendric structure of the MST graph with a hierarchy of nodes such that the nodes which have in terms of $d_{X Y}^{B}$ many close neighbours are higher in the hierarchy than the ones which are rather isolated. Although MST is only a specific choice of the network representation out of a broad spectrum of possible graphs, its usefulness in expressing the core information on the network structure has been shown in literature $[8,6,9,3]$. Using MST will make the graphical representation of our networks much more readable.

\section{Results}

In many models of the financial data, the returns are decomposed into two independent components expressed by a product of signed and unsigned stochastic processes. Typically, the former describes the fast changes of the signal and the latter corresponds to slow variations of volatility. Sometimes the statistical properties of these processes are even more different, as it is in the Mandelbrot multifractal model of asset returns, where the signed process is monofractal, while the unsigned one is multifractal. Such decomposition of the artificial data motivates us to consider a similar decomposition of the empirical data. In this case the slowly variable unsigned process could be the time-averaged amplitude of the returns (e.g. in windows of a given length). In the present study, however, such averaging should not be performed since our time series are too short. Due to this difficulty, instead of considering the fast and slow components, we prefer to study time series decomposed into their sign and amplitude parts

$$
g_{X}^{B}(i)=\operatorname{sgn}\left(g_{X}^{B}(i)\right)\left|g_{X}^{B}(i)\right|=s_{X}^{B}(i) a_{X}^{B}(i) .
$$

The signal $a_{X}^{B}$ is thus the instantaneous volatility instead the time-averaged volatility. We ask whether the networks based on such components share the same properties as the networks based on the original data. In other words, we ask whether the sign and the amplitude components share the same information on the currency market as do the complete signals.

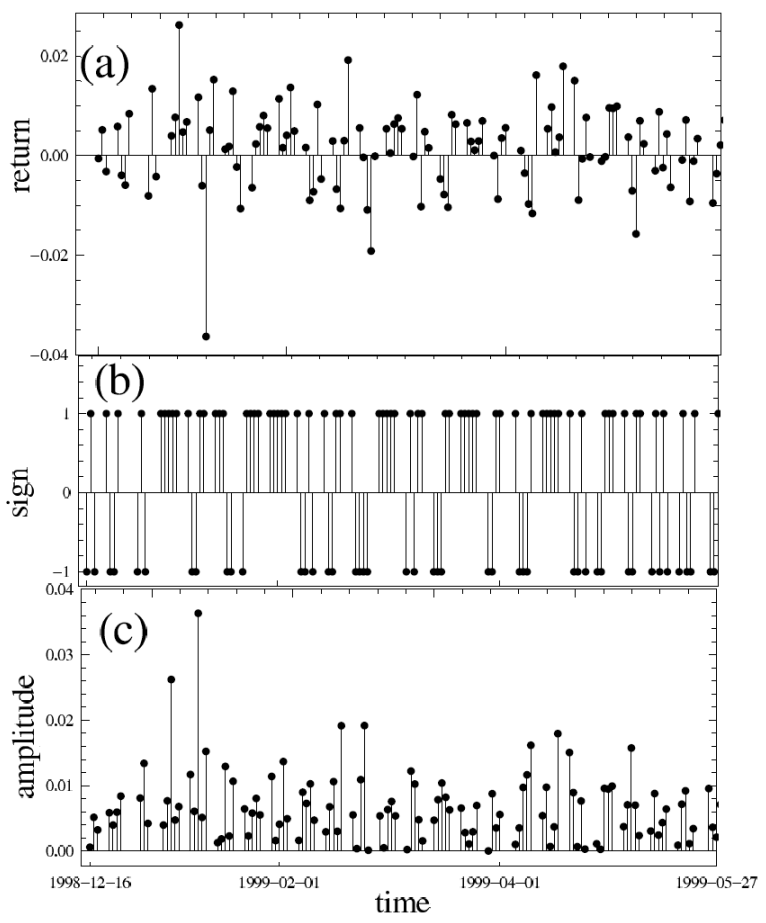

Fig. 1. Original time series of returns (a) decomposed into sign (b) and amplitude components (c). Gaps correspond to non-trading days.

In Fig. 1 we show the exemplary original signal and its components. We start our presentation with the choice $\mathrm{XAU}$ (gold) as the base currency. Gold, although at present cannot be considered a proper currency, is historically related to the currency market and its price evolution offers a convenient, independent reference frame for the whole market. MSTs for the XAU-based network are shown as weighted graphs in Fig. 2. Feature which is most striking is that for both types of signals all the internode connections have high weights. This is a manifestation of the fact that gold price is largely decoupled from the currency market, which in this representation acts as a large global cluster. At a more detailed level, the structure of internode connections among the nodes shows two prominent local clusters concentrated around USD and EUR, with the former clearly with a higher centrality than the latter. This picture remains qualitatively similar for the sign and the amplitude except for some minor restructuring of peripheral branches (which is a noise-like effect). This similarity together with the heavy weights suggest that gold price fluctuations have the same direction and magnitude if expressed in almost any currency. In other words, the gold price fluctuations are stronger (on average) than the fluctuations of all the exchange rates between currencies. This is an anticipated result. 

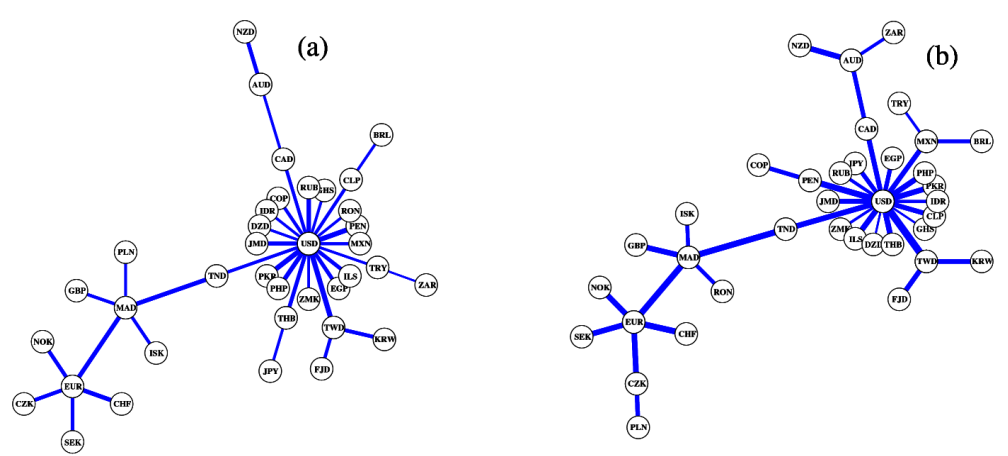

Fig. 2. Weighted MST graphs for the XAU-based network. The original signals of returns are decomposed into (a) signs, (b) amplitudes. Width of internode connections is proportional to connection weights.
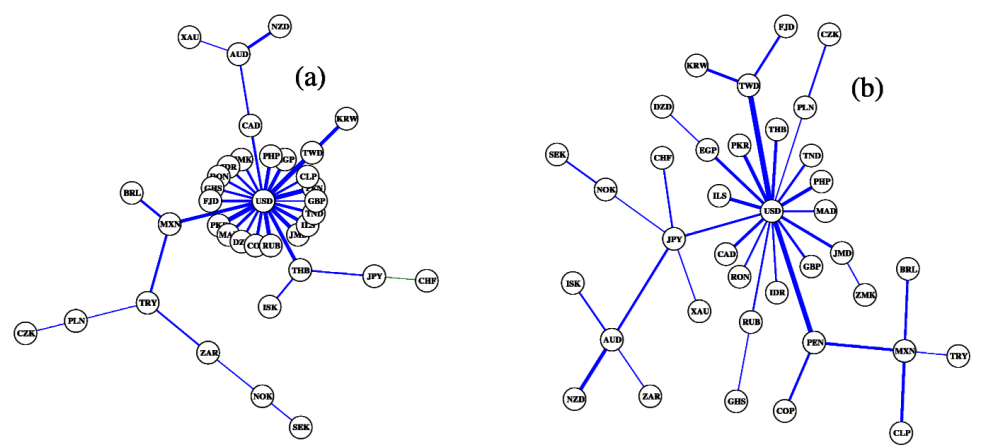

Fig. 3. Weighted MST graphs for the EUR-based network: (a) signs, (b) amplitudes.
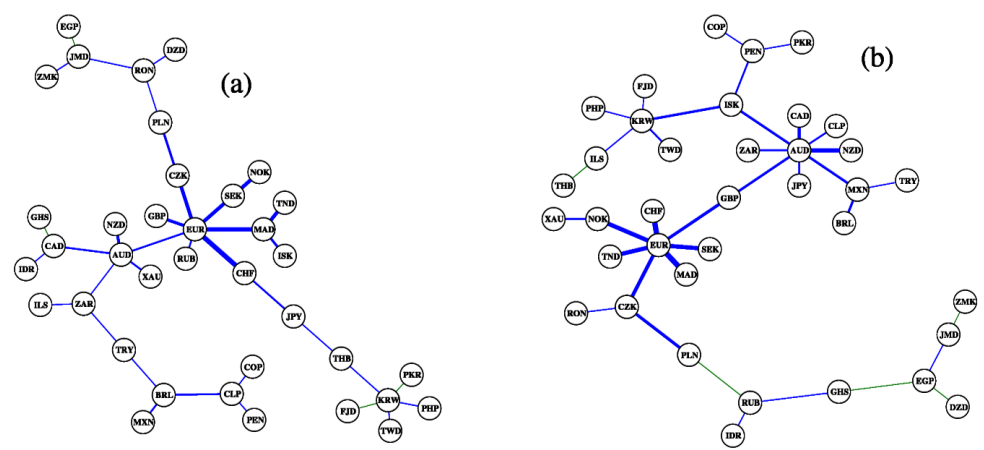

Fig. 4. Weighted MST graphs for the USD-based network: (a) signs, (b) amplitudes.

The EUR-based networks for signs (a) and amplitudes (b) is presented in Fig. 3. We see that although average connection weights are much smaller than in the previous case of XAU-based network, the structure is to a large extent similar. There is also no prominent node reshuffling between the sign-based and the-amplitude based representations. USD occupies the central position in both MSTs with slightly more links in the case of signs (17 vs. 21). Average weights are comparable in both cases.

Let us now look at the MSTs as viewed from the USD perspective (USD is the base currency, Fig. 4). Such networks are expected to be least coupled and large group of nodes behave as if the network was random. Figure 4 shows both the amplitude-based and the sign-based trees, which, as in Figs. 2 and 3, do not present any meaningful differences between their structures. In both graphs EUR plays a role of the main hub with the same multiplicity, attracting all other European currencies as well as MAD and TND, which are in the EUR basin of attraction. The secondary hub, AUD, is also prominent with comparable number of links as EUR, but with smaller weights. For both types of signals, it attracts its standard neighbours: NZD, CAD, ZAR, and XAU. However, for the amplitude signals connections are stronger than for the sign ones. AUD also attracts more nodes in the case of amplitude signals among which notable examples are MXN and ISK which are not connected with this node if signed time series are considered (both signs and returns). It is worth 
noting that for both types of signals (as well as for the complete signals discussed in [3]) AUD reveals largely independent dynamics, different from both USD and EUR. This is expressed by the fact that, in MSTs, its closest neighbours are some other, peripheral currencies and the corresponding edges have small weights. (A curious placement of ISK in this and other trees in Figs. 2-4, contrary to its expected relation to EUR, may originate from the financial crisis which took place in Iceland in 2008, which led this currency to devaluation and its consequent decoupling from the EUR-based cluster, observed even in the 10-year-long signals.)

As regards the secondary cluster structure, especially the South-East Asian and the Latin American cluster, it occurs that they are based on signs, at least to some extent, which is not the case for the strongest clusters of European and commodity-related currencies. In general, it is observed that for the USD-based network the amplitude signals do not develop so clear regional cluster structure as the signed signals.

Let us now consider the MST topology in a quantitative manner by means of the characteristic path length $L^{B}$ given by the formula

$$
L^{B}=\frac{1}{N(N-1)} \sum_{X, Y: X \neq Y} l^{B}(X, Y),
$$

where $l^{B}(X, Y)$ stands for length of the path connecting nodes $X$ and $Y$. Characteristic path length measures how compact is the tree in a given network representation $B$ : low values of $L^{B}$ describe a compact MST with short connection paths between any pair of nodes, while high values of $L^{B}$ correspond to a "stretched" tree with long branches. A stretched structure of a Forex-based MST would thus mean that there is no node attracting a significant number of other nodes and that a typical node have a few closest neighbours. In contrast, a compact MST would be related to the existence of one or at most a few central nodes with many connected satellites; a typical node would be a satellite of a central node and would therefore have only one neighbour. From a point of view of the base currency $B$, a high value of $L_{B}$ and, at the same time, small values of $L_{B^{\prime}}$ for many other base currencies $B^{\prime}$, can suggest that the currency $B$ constitutes a high-degree node in the $B^{\prime}$-based trees; removing this node from the network by selecting the related currency to be the base gives freedom to its satellites and stretches MST.

TABLE

The characteristic path length $L^{B}$ and average weighted clustering coefficient $\widetilde{C}^{B}$ for a few representative choices of the base currency $B$ calculated for the full period 1999-2008 and deconstruct the real data into sign and amplitude components.

\begin{tabular}{l|c|c|c|c|c|c|c|c|c}
\hline \hline \multicolumn{1}{c|}{ Base: } & CHF & CZK & EUR & GBP & GHS & JPY & PLN & USD & XAU \\
\hline$L_{\text {(return) }}^{B}$ & 1.63 & 1.73 & 1.53 & 2.33 & 1.55 & 1.95 & 1.99 & 4.10 & 1.65 \\
$L_{\text {(sign) }}^{B}$ & 1.95 & 1.77 & 1.72 & 2.12 & 2.02 & 1.85 & 1.79 & 4.25 & 1.86 \\
$L_{(\text {abs) }}^{B}$ & 1.59 & 1.67 & 1.60 & 1.93 & 1.96 & 1.69 & 1.67 & 4.13 & 1.64 \\
$\widetilde{C}_{(\text {return) }}^{B}$ & 0.431 & 0.202 & 0.333 & 0.311 & 0.929 & 0.512 & 0.511 & 0.111 & 0.712 \\
$\widetilde{C}_{(\text {sign) }}^{B}$ & 0.358 & 0.181 & 0.312 & 0.329 & 0.919 & 0.391 & 0.371 & 0.139 & 0.911 \\
$\widetilde{C}_{(\text {abs })}^{B}$ & 0.326 & 0.175 & 0.309 & 0.308 & 0.921 & 0.377 & 0.389 & 0.139 & 0.771
\end{tabular}

Results for our data are shown in Table for a few base currencies. On average, the most distributed MSTs (largest $L^{B}$ ) are formed by the signs, while the original signals (returns) and their absolute values are characterized by more compact MSTs. As expected, the longest paths characterize the USD-based trees which reflects the least correlated nature of this representation of the currency network. On the opposite end are the EUR-based trees. This is also understandable due to the fact that if EUR is chosen to be the base, there remains only one hub (USD) which makes the network highly centralized (Fig. 3).

Another measure which can provide us with some insight into the network topology is the weighted clustering coefficient. It is defined for a complete network as [10]:

$$
\widetilde{C}^{B}=\frac{1}{N} \sum_{X} \widetilde{c}^{B}(X),
$$

where

$$
\begin{aligned}
& \widetilde{c}^{B}(X)=\frac{1}{k_{X}^{B}\left(k_{X}^{B}-1\right)} \sum_{Y, Z}\left(\widetilde{\omega}_{X Y}^{B} \widetilde{\omega}_{Y Z}^{B} \widetilde{\omega}_{Z X}^{B}\right)^{\frac{1}{3}}, \\
& \widetilde{\omega}_{P Q}^{B}=\frac{\omega_{P Q}^{B}}{\max _{P Q}\left[\omega_{P Q}^{B}\right]} .
\end{aligned}
$$

In this case $k_{X}^{B}$ is the degree of node $X$. The correlation coefficient, which can be defined for both binary and weighted networks, assumes non-zero values if the network comprises many triangles. A triangle is a motif 

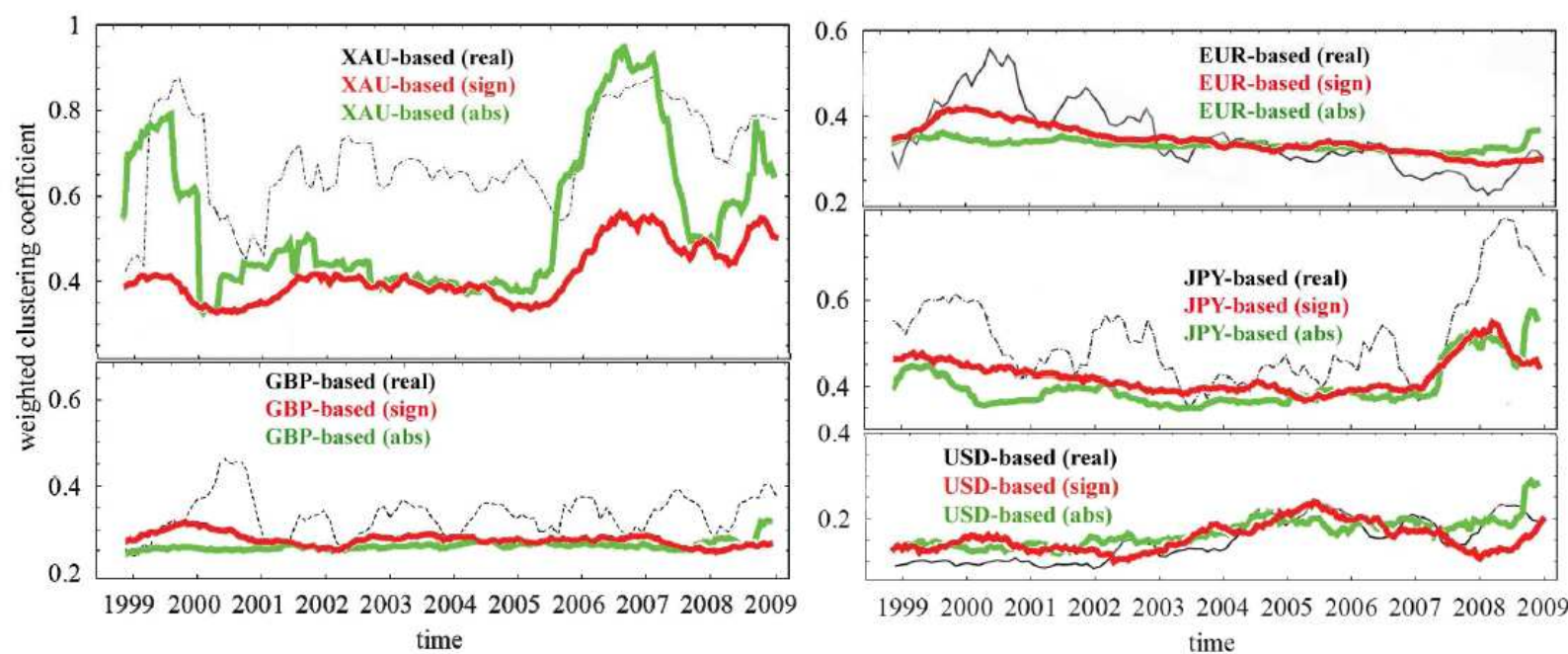

Fig. 5. Weighted clustering coefficient $\widetilde{C}^{B}(t)$ for a few exemplary choices of the base currency. In each part three types of data are presented: original signals (dashed black), signs (heavy red), and amplitudes (heavy green).

characterized by the following condition: if a node $i$ is connected to a node $j$ and to a node $k$, this implies that the nodes $j$ and $k$ are also mutually connected. For the weighted networks, in order to take the weights into consideration, this definition can be altered according to Eqs. (8) and (9). Thus, the weighted clustering coefficient assume high values if the above condition is fulfilled but additionally all the triangle edges have high weights.

Numerical values of $\widetilde{C}^{B}$ are collected in Table for the same base currencies as before. The results are highly $B$-dependent. For CHF, EUR and other continental European currencies, the clustering coefficient is highest for the original signals and lowest for the amplitudes, with the difference being largest for CHF and PLN. The same refers to JPY. In contrast, for the XAU-based and the GBP-based networks the highest clustering is observed for the signs, while both the amplitudes and the returns are significantly less clustered. USD is somehow peculiar in this respect, with equal level of clustering for the signs and the amplitudes, and a smaller level of clustering is observed for the returns.

We also consider the temporal stability of $\widetilde{C}^{B}$ by using a moving window of 120 trading days (approximately 6 months). The results are presented in Fig. 5 for 4 base currencies and XAU. The most interesting observation is that for some choices of $B$, the network topolgy is much more stable in the case of the decomposed signals (the signs and the amplitudes) than in the case of original returns.

This is true for GBP, JPY, and EUR, but not true for XAU and USD. These deconstructed signals exhibit sometimes different trends that the original complete signals, as it can be seen, e.g., in the case of the USD-based network. Nevertheless, in the case of the signs and the amplitudes, the EUR-based network exhibits a clear downward trend, similar to the one for the returns, indicating that the network viewed from the EUR perspective

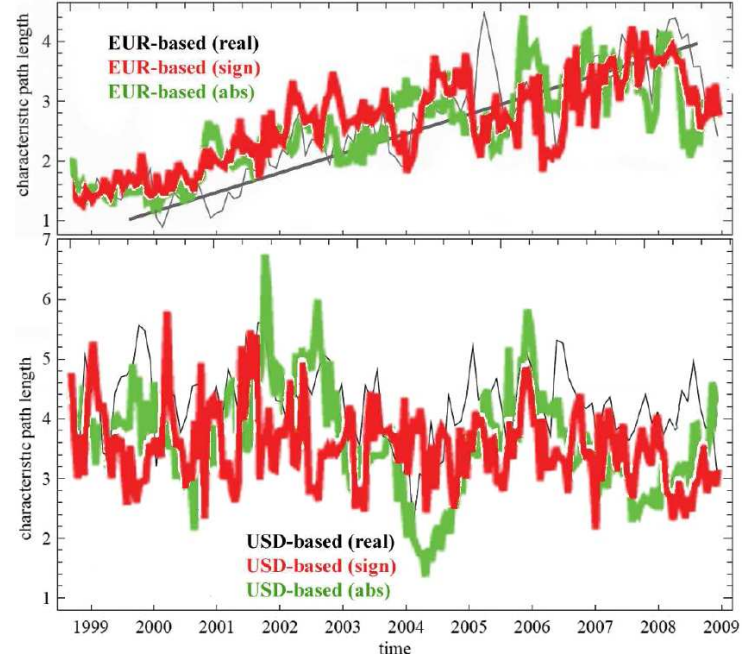

Fig. 6. Characteristic path length $L^{B}(t)$ for the EUR-based and the USD-based network representations. Three types of data are presented: original signals (thin black), signs (heavy red), and amplitudes (heavy green). Black solid line indicates a linear trend in $L^{\mathrm{EUR}}(t)$ for the returns.

becomes more random-like (small clustering) than in the early years of EUR (Fig. 6). This observation can be directly verified by looking at Figs. 7 and 8 , in which MST graphs calculated in two-years-long windows are shown (signs in Fig. 7 and amplitudes in Fig. 8). These results can be considered another manifestation of an increasing role of euro in the world trading system $[1,3]$. 

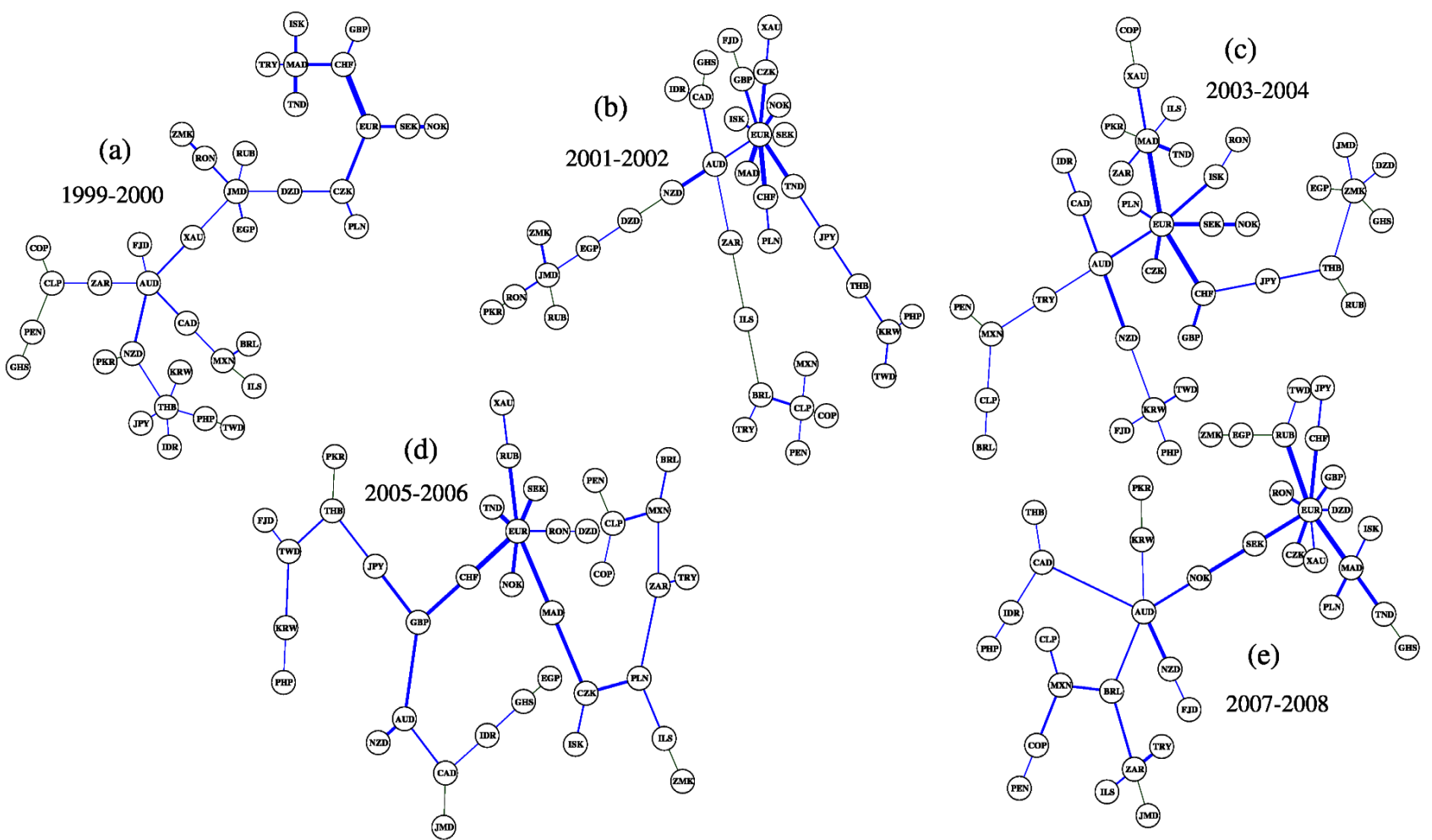

Fig. 7. Minimal spanning trees for the USD-based network calculated for the sign time series in subintervals of 2 years (a)-(e). Green edges connect anticorrelated nodes.

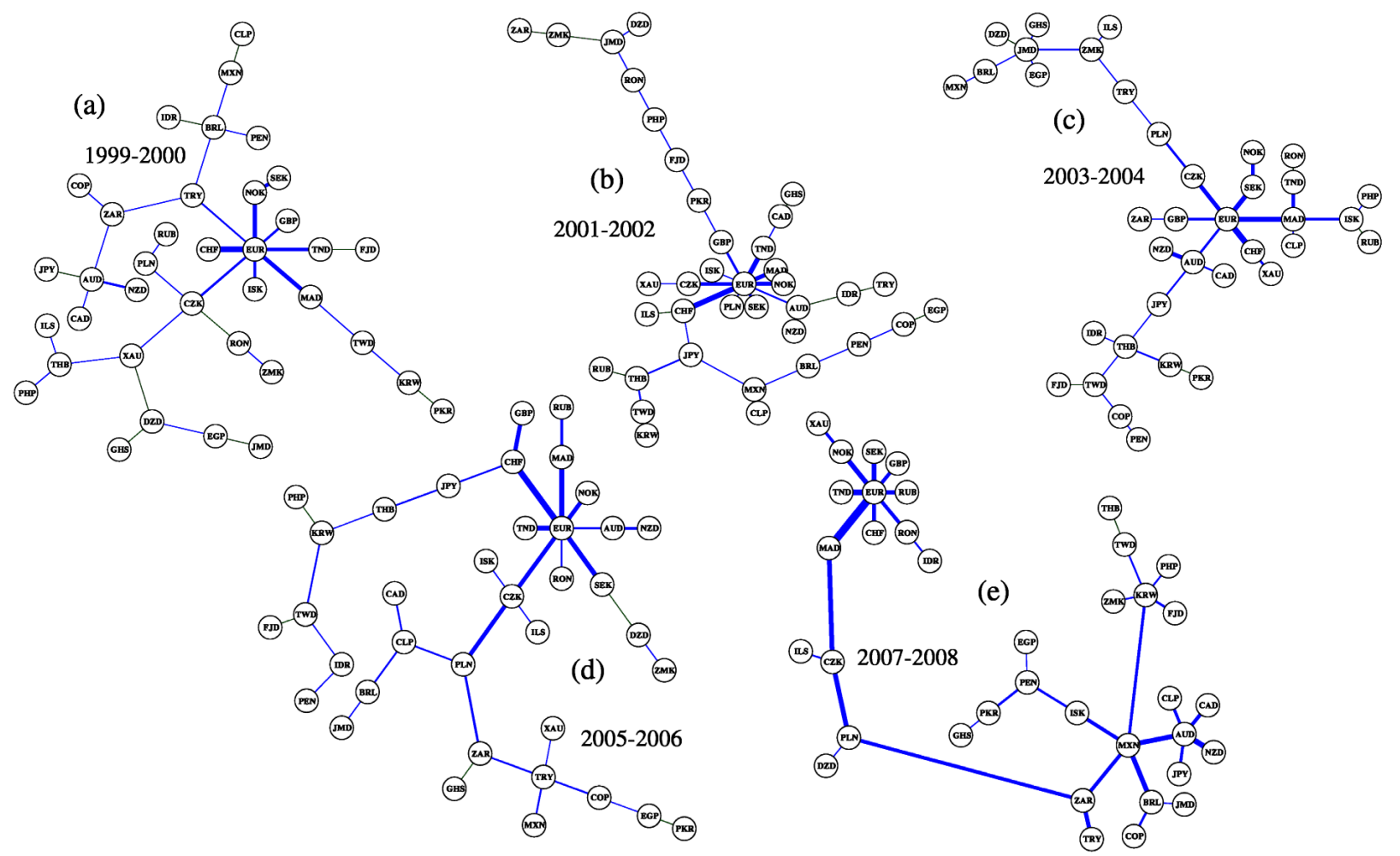

Fig. 8. Minimal spanning trees for the USD-based network calculated for the amplitude time series in subintervals of 2 years $(\mathrm{a})-(\mathrm{e})$. 


\section{Conclusions}

In summary, we studied the time series of currency exchange returns decomposed into their sign and amplitude components. We showed that the MST graphical structure does not change considerably for the components if compared with the original signals. However, more precise quantitative tools such as the characteristic path length and the weighted clustering coefficient indicate some restructuring of nodes and connection weights. We also observed that temporal stability of the networks for the components is better than for the returns. These are preliminary conclusions and more work is needed in order to better understand their significance.

\section{References}

[1] S. Drożdż, A.Z. Górski, J. Kwapień, Eur. Phys. J. B 58, 499 (2007)
[2] A.Z. Górski, S. Drożdż, J. Kwapień, Eur. Phys. J. B 66, 91 (2008).

[3] J. Kwapień, S. Gworek, S. Drożdż, Acta Phys. Pol. B 40, 175 (2009).

[4] J. Kwapień, S. Gworek, S. Drożdż, A.Z. Górski, J. Econ. Interact. Coord. 4, 55 (2009).

[5] Sauder School of Business, Pacific Exchange Rate System, http://fx.sauder.ubc.ca/data.html .

[6] M. McDonald, O. Suleman, S. Williams, S. Howison, N.F. Johnson, Phys. Rev. E 72, 046106 (2005).

[7] R.N. Mantegna, Eur. Phys. J. B 11, 193 (1999).

[8] G. Bonanno, G. Caldarelli, F. Lillo, C. Miccichè, N. Vandewalle, R.N. Mantegna, Eur. Phys. J. B 38, 363 (2004).

[9] P. Sieczka, J.A. Hołyst, Physica A 388, 1621 (2009).

[10] J.P. Onnela, J. Saramaki, J. Kertesz, K. Kaski, Phys. Rev. E 71, 065103 (2005). 